\title{
Paracoccidioidomicose sistêmica com envolvimento do sistema nervoso central
}

\author{
Systemic paracoccidioidomycosis with central nervous system involvement
}

\author{
Antônio Luiz Wiener Pureza Duarte, Giovanni Baruffa, Helena Beatriz Grassi \\ Terra, Décio Valente Renck, Daniela de Moura e Cunha Petrucci
}

\begin{abstract}
Resumo É relatado o caso de um paciente portador de paracoccidioidomicose sistêmica, com comprometimento oral e ganglionar regional e posterior envolvimento pulmonar. O paciente, tratado com drogas específicas(anfotericina $B$, itraconazol, sulfametoxazol + trimetoprim) e acompanhado durante seis anos, foi ao óbito com extenso comprometimento do sistema nervoso central
\end{abstract}

Palavras-chaves: Paracoccidioidomicose. Sistema nervoso central.

Abstract A clinical case of a patient bearing systemic paracoccidioidomycosis with regional ganglionic and oral exposure and later pulmonary envolvement is presented. The patient was treated with especific drugs (amphotericin B, itraconazole, sulfamethoxazole-trimethoprim) and followed throughout a 6-year period and eventually died showing an extensive envolvement of the central nervous system.

Key-words: Paracoccidioidomycosis. Central nervous system.

Paracoccidioidomicose (PCM), infecção fúngica causada pelo Paracoccidioides brasiliensis é doença sistêmica de grande prevalência na América Latina, sendo endêmica no Brasil, Argentina, Venezuela e Colômbia11.

A PCM é adquirida, habitualmente, por via inalatória e é encontrada principalmente em adultos, mais no sexo masculino $(10,7: 1)$, com atividades ligadas à agricultura ou a outras lides rurais, nos quais pode configurar doença ocupacional12. A penetração do $P$. brasiliensis pelas mucosas (orofaríngea, intestinal, retal) e através da pele seria excepcional11.

O envolvimento do sistema nervoso central ocorre em cerca de $10 \%$ dos casos e se apresenta sob duas formas: a) a forma meníngea, com inflamação da leptomeninge, predominantemente na base do crânio, originando uma leptomeningite similar àquela tuberculosa; b) a forma granulomatosa, também conhecida como forma pseudotumoral, que se apresenta com granulomas intraparenquimatosos, únicos ou múltiplos, de localização habitualmente supratentorial10 16.

A PCM é endêmica no Rio Grande do Sul, sendo que a maioria dos casos conhecidos é oriunda da metade norte do estado4.

Na metade sul, os casos publicados não chegam a três dezenas e procedem da Serra e da Encosta do Sudeste ${ }^{3}$. Não temos conhecimento do envolvimento do SNC no Rio Grande do Sul. Esse aspecto, o longo período de observação do paciente e a falência da terapêutica antifúngica empregada motivam o relato a seguir.

Santa Casa de Misericórdia de Pelotas, Pelotas, RS.

Endereço para correspondência: Prof. Antônio Luiz W.P. Duarte. Av. Dom Joaquim 910, 96020-260 Pelotas, RS Tel: 5553 223-2065; 5553 982-3482; Fax: 5553 222-1616

Recebido para publicação em 4/8/98. 


\section{RELATO DO CASO}

JPB, 37 anos, masculino, branco, trabalhador rural, natural de Piratini, RS, município localizado no Planalto Uruguaio-Rio-Grandense (coxilha e serra). O paciente relatou ser portador de paracoccidioidomicose desde 1992. A doença teria iniciado na cavidade oral, sem comprometimento pulmonar aparente. Na época foi tratado com anfotericina $B$, na dose total de $35 \mathrm{mg} / \mathrm{kg}$. Decorridos 2 anos, o paciente volta ao hospital com dispnéia, febre, tosse produtiva com escarro hemoptóico, adenomegalias cervicais e queda do estado geral. A biópsia de um linfonodo cervical evidenciou Paracoccidioides brasiliensis, sendo, então, internado para tratamento clínico. O exame físico, na internação, mostrava emagrecimento e murmúrio vesicular rude à ausculta pulmonar. O laboratório registrava uma leucocitose (18.100 leucócitos $/ \mathrm{mm}^{3}$ ) com desvio à esquerda ( $5 \%$ de bastões) e uma VSG de $66 \mathrm{~mm}$ na $1 \underline{\underline{a}}$ hora. A radiografia torácica era compatível com a doença em questão. Fez novo tratamento com anfotericina $B$, na dose total de $2 \mathrm{~g}$ $(35 \mathrm{mg} / \mathrm{kg})$, não obtendo melhora. Em junho de 1995, um tratamento com itraconazol (200mg/dia), por um período de 8 meses, resultou em aparente cura clínica e radiológica.

Em 12.03.97, o paciente reinterna com tosse seca e radiografia torácica compatível com reativação da doença. Foi tratado com itraconazol ( $200 \mathrm{mg} / \mathrm{dia})$ e sulfametoxazol-trimetoprim ( $2400 \mathrm{mg} /$ dia de sulfa e $480 \mathrm{mg} /$ dia de trimetoprim). Obteve alta hospitalar em 05.04.97 para continuar o tratamento em seu domicílio.

Em 23.03.98, o paciente, ainda em tratamento com o esquema acima, retornou com queixa de hemoptise após esforço físico, cefaléia e febre, sendo então internado.

$\mathrm{Na}$ baixa, o paciente apresentava-se emagrecido, corado, hidratado, anictérico, eupneico, lúcido, orientado, apresentando episódios de febre que chegavam a $39^{\circ} \mathrm{C}$. O exame físico não demonstrou alterações significativas.
Uma tomografia computadorizada de tórax foi realizada mostrando presença de áreas em vidro fosco, bilateralmente, caracterizando a atividade da doença em questão.

03.04.98 - Anti HIV: não reagente.

O paciente mantinha o mesmo quadro febril, apesar de estar usando SMZ + TMP endovenoso, na dose de $2400 \mathrm{mg} /$ dia de $\mathrm{SMZ}$ e $480 \mathrm{mg} / \mathrm{dia}$ de TMP.

Sem alteração significativa ao exame físico, apresentou um quadro de agitação psicomotora, desorientação, cefaléia intensa e vômitos após duas semanas de internação. O exame neurológico mantinha-se normal, sem nistagmo, Babinski ou sinais meníngeos. Exame de fundo de olho normal.

Foi solicitada fibrobroncoscopia e lavado bronco-alveolar e feito exame micológico a fresco onde foram observadas células arredondadas, de dupla parede, refringentes, com e sem gemulação, compatíveis com $P$. brasiliensis. $\mathrm{O}$ material foi corado pela técnica de Grocott e houve impregnação do fungo pela prata, mostrando nitidamente os múltiplos brotamentos em torno da célula-mãe com o aspecto característico de roda de leme.

Em 12.04.98, reiniciou-se anfotericina B.

Uma tomografia computadorizada cerebral (TCC), realizada em 13.04.98, mostrou presença de múltiplas áreas hiperdensas, arredondadas, algumas apresentando calcificações periféricas, sem realce significativo pelo meio de contraste e sem edema adjacente nas lojas supra e infratentoriais. Conclusão: achados compatíveis com paracoccidioidomicose encefálica (Figuras 1 e 2).

Em 23.04.98, com um total de $400 \mathrm{mg}$ de anfotericina, apareceram dispnéia intensa, cianose e estertoração pulmonar, evoluindo para edema agudo de pulmão. Foram tomadas as medidas terapêuticas para o quadro clínico, mas não houve resposta, e o paciente foi ao óbito em 24.04.98.

\section{DISCUSSÃO}

O paciente procedia de um município da Serra do Sudeste, recortado por riachos e córregos, com solos férteis e restos de mata nativa. O sexo, a idade e a profissão do paciente correspondem aos habitualmente encontrados na PCM.
A evolução do quadro, com aparente início na mucosa oral, o comprometimento extenso e grave do aparelho respiratório e a sucessiva disseminação ao SNC estavam dentro das possibilidades evolutivas da PCM. 

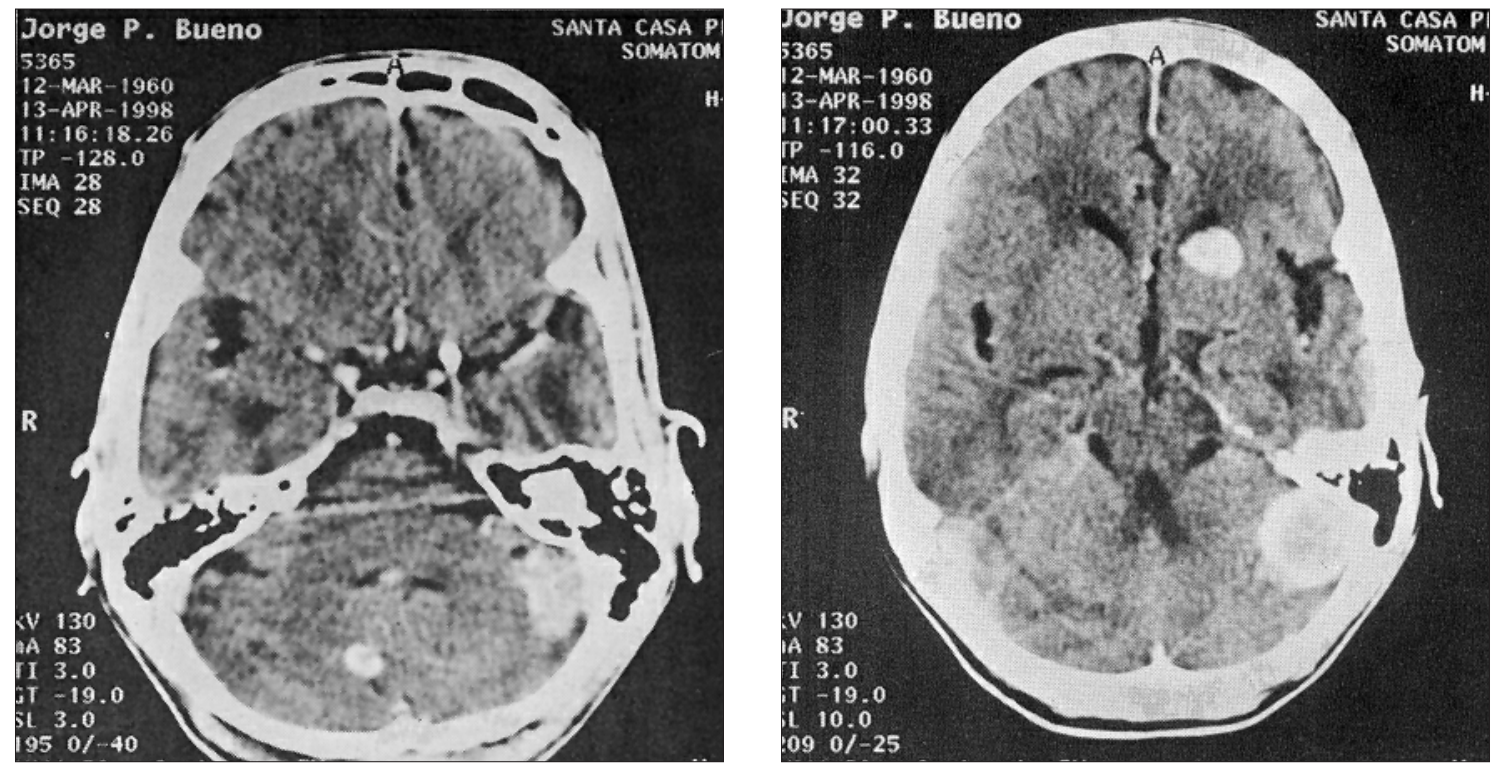

Figuras 1 e 2 - TC cerebral mostrando presença de múltiplas áreas hiperdensas, arredondadas, algumas com calcificações periféricas, compatíveis com paracoccidioidomicose encefálica.

O acompanhamento do paciente durante 6 anos pelos mesmos profissionais permitiu observar o curso clínico da micose que, aparentemente, respondeu pouco ao tratamento específico. Esse tratamento, além de não propiciar a cura, não conseguiu impedir a propagação da infecção ao $\mathrm{SNC}$, resultando disso um quadro pseudotumoral e a morte do paciente.

A TCC documentou a distribuição dos granulomas no cérebro, em localização supratentorial, como vimos, bastante característica do envolvimento do SNC nesta patologia. Este exame, aliado aos dados clínicos e laboratoriais, é altamente sugestivo de PCM, apesar de não ser diagnóstico.

Surpreendeu-nos a relativa resistência do $P$. brasiliensis aos antifúngicos empregados: anfotericina B, itraconazol e SMZ-TMP, apesar de as doses terem sido aquelas indicadas e a administração ter coberto por inteiro os períodos de hospitalização, com recomendação ao paciente de não descontinuá-la nos períodos de alta. Achamos improvável que o paciente tenha interrompido o tratamento durante os períodos de alta hospitalar. Tanto o paciente quanto os seus familiares foram diligentes em procurar os médicos todas as vezes em que os fármacos estavam por terminar, recebendo amostras previamente solicitadas aos laboratórios. Infelizmente não foi testada a situação imunológica do paciente com paracoccidioidina intradérmica, por isso não é possível concluir se o fracasso terapêutico foi devido à resistência aos fármacos específicos ou à falência imunológica do paciente.

Pelas consultas bibliográficas, seria este o primeiro caso de PCM com envolvimento do sistema nervoso central no Rio Grande do Sul.

\section{REFERÊNCIAS BIBLIOGRÁFICAS}

1. Arathoon EG, Brummer E, Stevens DA. Efficacy of Itraconazole in Blastomycosis in Murine Model and Comparison with Ketoconazole. Mycosis 32:109-112, 1989.

2. Araujo JC, Werneck L. South American Blastomycosis as a Posterior Fossa Tumor. Journal Neurosurg 425-428, 1978.

3. Baruffa G. Blastomycose Sud-Américaine dans la Región Sud du Rio Grande do Sul, Brésil. VII Congrès
International de Parasitologie. Bulletin de la Société Française de Parasitologie 8:376, 1990.

4. Bopp C, Bernardi CD. Geopatologia da Blastomicose Sul-Americana. Hospital 71:1613-1630, 1967.

5. Costa PDDA, Oliveira NCDE, Picanco M. Blastomicose Sul-Americana. Forma Ganglionar. Hospital 77:805-817, 1970. 
6. Gonçalves AJR, Figueiredo PC, Barbosa LSG, Aguilar J, Almeida SL, Andrade EM, Santos FB, Pedrosa MC, Nunes R. Paracoccidioidomicose. Considerações sobre algumas formas incomuns. Arquivos Brasileiros de Medicina 58:301-308, 1984.

7. Guerreiro CAM, Chuluc SSD, Branchini MLN. A New Treatment For Large Cerebral Paracoccidioidomycosis. Arquivos de Neuro-Psiquiatria 45:419-423, 1987.

8. Hutzler RU, Brussi MLP, Capitani C. Acometimento Neurológico da Paracoccidioidomicose. Revista Paulista de Medicina 103:243-244, 1985.

9. Kauffman CA. Newer Developments in Therapy for Endemic Mycosis. Clinical Infectious Disease 19:28-32, 1994.

10. Magalhães ACA, Bacheschi LA, Caramelli $P$, Lo LSS, Neto JRM, Yasuda MAS, Magalhães A. Paracoccidioidomicose do Sistema Nervoso Central: Estudo de Cinco Casos por Ressonância Magnética. Revista do Hospital das Clínicas da Faculdade de Medicina da Universidade de São Paulo 48:94-97, 1993.
11. Martinez R, Ferreira MS, Mendes RP, del Negro GB, Gonçalves EG, Telles Filho FQ. In: Veronesi R, Focaccia R (eds) Tratado de Infectologia, 1a edição, Atheneu, São Paulo, p. 1081-1111, 1997.

12. Morato-Fernandez RN, Beraldo PSS, Masini M, Costa PHC. Paracoccidioidomicose de Localização Intramedular e cerebral. Arquivos de Neuro-Psiquiatria 49:192-197, 1991.

13. Nóbrega JPS. Micoses do Sistema Nervoso Central. Arquivos Brasileiros de Neurocirurgia 1:157-171, 1982.

14. Rodacki MA, De Toni G, Borba LA, Oliveira GG. Paracoccidioidomycosis of the Central Nervous System: CT Finding. Neuroradiology 37:636-641, 1995.

15. Sarosi GA, Davies SF. Therapy for Fungal Infections. Mayo Clinic Proceedings 69: 1111-1117, 1994.

16. Teive HAG, Arruda WO, Ramina R, Meneses MS, BleggiTorres LF, Telles-Filho FQ Paracoccidioidomycosis Granuloma Simulating Posterior Fossa Tumor. Journal of the Royal Society of Medicine 84: 562-563, 1991. 